\title{
Action Spaces for Efficient Bayesian Tracking of Human Motion
}

\author{
${ }^{\dagger}$ Ignasi Rius, ${ }^{\ddagger}$ Javier Varona, ${ }^{\S}$ Jordi Gonzàlez, ${ }^{\dagger} J u a n$ J. Villanueva
}

${ }^{\dagger}$ Centre de Visió per Computador

Edifici O. Campus UAB. 08193, Bellaterra, Spain. irius@cvc.uab.es

${ }^{\ddagger}$ Dept. de C.Mat. i Informàtica, UIB, Mallorca, Spain

$\S$ Institut de Robòtica i Informàtica Industrial, CSIC-UPC, Barcelona, Spain

\begin{abstract}
Bayesian tracking implemented as a particle filter is one of the most used techniques for full-body human tracking. However, given the high-dimensionality of the models to be tracked, the number of required particles to properly populate the space of solutions make the problem computationally very expensive. To overcome this, we present an efficient scheme which makes use of an action model that guides the prediction step of the particle filter. In this manner, particles are propagated to locations in the search space with most a posteriori information. Hence, we sample from a smooth motion model only those postures which are feasible given a particular action. We show, that this scheme improves the efficiency and accuracy of the overall tracking approach.
\end{abstract}

\section{Introduction}

Automatic full human body tracking remains as one of the most challenging problems from visual human motion analysis. Tracking full-body 3D human poses from a monocular sequence of $2 \mathrm{D}$ images implies the use of very high dimensional representations and non-linear models. To overcome these issues, many approaches make use of Bayesian filtering techniques. This probabilistic framework can deal with multiple hypotheses, and brings a principled way to incorporate a priori knowledge about human motion into the tracking, so the solution space can be explored in a more efficient manner. Usually, particle filters are used to implement such a framework.

Particle filters were first introduced in computer vision by Isard et al. in CONDENSATION for shape tracking [4]. They showed that particle filters supply a powerful tool for representing and propagating complex posterior distributions at the expense of a high computational cost. The number of needed particles grows exponentially as the number of dimensions to be tracked does [5]. This fact is clearly visible in human motion tracking, due to the high DOF needed to represent human postures. For this reason, it is necessary to make particle filters more efficient. For example, the annealed particle filter aims to reduce the number of required samples by successively pruning less likely hypotheses [1]. Alternatively, it is possible to use efficient motion models which concentrate particles in areas of interest. For instance, in [8] they used a cyclic dynamic model of the walking action for full human body tracking. Likewise, Ning. et al [6] tracked a walking sequence of a 12 DOF body model using particle filtering and another dynamic model of walking, which included constraints on human motion, and learnt the parameters of motion per each joint from examples. In [9], Sidenbladh et al. generalized their approach to include different actions than walking. They learnt the dynamic model from a prerecorded set of human motions, and predictions were made assuming a Gaussian distribution over subsequences of the learned motions. As a result, particle wastage was avoided by concentrating particles in areas where motion was observed before. However, the model can only predict postures which were present in the motion database.

Our approach makes use of particle filtering to track a 37 DOF human body model, and uses the aspace action model [2]. This model focuses and restricts the search space only to feasible human postures within a particular action. Thus, by considering representative training sets for each action, we result in an action model specific enough to avoid particle wastage, but general enough to track any performance of an action. As a result, we explore the space of solutions in an efficient manner, reducing the number of needed particles to successfully track fullbody $3 \mathrm{D}$ human motion. Section 2 presents the tracking 
framework. Section 3 reviews the building of the action model. Section 4 details the integration of the action model into the tracking framework. In Section 5, results of the tracking are presented for a sequence of a bending action not considered in the training set.

\section{Tracking framework}

Our aim is to recursively estimate the configuration of a 3D human body model, given the available measurements up to each moment, i.e. the 2D image sequence. The Bayesian filter recursively estimates the state of the tracked object at each time step given the evidences (image data) up to that moment. It decomposes the problem in two differentiated steps, i.e. the prediction and update steps. The prediction step projects forward the model parameters to the next time step by means of a temporal prior. Then the update step makes use of a likelihood probability function in order to evaluate the fitness of the predictions to the evidences available at each moment.

Formally, within the Bayesian filtering framework, we formulate the computation of the posterior distribution $p\left(\phi_{t} \mid \mathbf{I}_{t}\right)$ of our model parameters over time as follows:

$$
p\left(\phi_{t} \mid \mathbf{I}_{t}\right) \propto p\left(I_{t} \mid \phi_{t}\right) \int p\left(\phi_{t} \mid \phi_{t-1}\right) p\left(\phi_{t-1} \mid \mathbf{I}_{t-1}\right) d \phi_{t-1},
$$

where $\phi_{t}$ represents a particular pose of the human body at time $t, \mathbf{I}_{t}$ is the image sequence up to time $t, p\left(I_{t} \mid \phi_{t}\right)$ is the likelihood of observing the image $I_{t}$ given the parametrization $\phi_{t}$ of our model at time $t$, and finally $p\left(\phi_{t} \mid \phi_{t-1}\right)$ is the dynamic model. Details are given in [7].

Unfortunately, an analytical solution of eq.(1) cannot be calculated unless strong assumptions about Gaussianity and linearity of the involved distributions are made. Instead, we use particle filtering techniques in order to approximate the true posterior pdf by means of a discrete weighted set of samples.

Hence, whilst the likelihood function decides which particles are worth to propagate, the dynamic model is responsible for guiding the exploration of the space of solutions. Thus, the performance and robustness of the overall tracking can be improved designing efficient search strategies. In other words, given the high-dimensionality present in human motion tracking, our predictions must be generic enough to track any motion, but specific and efficient enough to focus particles only to areas with high a posteriori information.

\section{Building the action model}

We use a 3D human body model composed of 12 limbs with 3 DOF per joint expressed as relative angles in a 3D polar coordinate system. A normalised height of the hip is also included in the model. By means of a commercial Motion Capture System, we acquired 45 performances, in average, of 9 different actions performed by 9 different actors. We may refer the reader to [7] for details on the body model and motion database used. The process for building the action model is summarized next.

Let $\phi$ be a 37-dimensional vector representing a particular human posture, and $\Phi$ be a sequence of human postures, hereafter performance. Then, for a particular action $A_{i}$, we compute PCA over all the training performances $\Phi_{j}$ for that action. The resulting PCA-like space - hereafter the aSpace - will be denoted as $\Omega^{A_{i}}$. The projections $\tilde{\Phi}_{j}$ on the aspace of $\Phi_{j}$ constitute a lower dimensional version from the original data.

Subsequently, we normalise the length of each performance by means of a cubic spline, and compute the mean performance $\overline{\mathbf{g}}^{A_{i}}$. Afterwards, a key-frame set $\vec{K}^{A_{i}}$ is found from $\overline{\mathbf{g}}^{A_{i}}$ by selecting the maximum and minimum distant postures from the mean posture in the same fashion than [3]. The found key-frames are used to synchronize all the performances from the training set. Hence, we can resample all the performances so they have the same number of frames between each key-frame, obtaining a synchronised version of the training set.

Subsequently, we learn the standard deviation $\sigma_{k}^{A_{i}}$ present in each frame $k$ of the synchronised training set for the action $A_{i}$. Finally, our action model is defined as:

$$
\Gamma^{A_{i}}=\left(\Omega^{A_{i}}, \hat{\mathbf{g}}^{A_{i}}, \sigma_{k}^{A_{i}}\right),
$$

where $\Omega^{A_{i}}$ defines the aSpace, $\hat{\mathbf{g}}^{A_{i}}$ stands for the synchronised version of the mean performance.

The learned action model can be used to probabilistically determine whether a posture belongs to action $A_{i}$ or not in the manner described in the next section.

\section{Using the action model}

The action model consists in a priori knowledge on human motion. Such information can be incorporated into the Bayesian tracking approach by means of the temporal prior. Hence, we reformulate Eq.(1) as

$$
p\left(\phi_{t} \mid \mathbf{I}_{t}\right) \propto p\left(I_{t} \mid \phi_{t}\right) \int p\left(\phi_{t} \mid \phi_{t-1}, \Gamma\right) \cdot p\left(\phi_{t-1} \mid \mathbf{I}_{t-1}\right) d \phi_{t-1} .
$$

Assuming independce between $\phi_{t-1}$ and $\Gamma$ we can decompose $p\left(\phi_{t} \mid \phi_{t-1}, \Gamma\right)$ as

$$
p\left(\phi_{t} \mid \phi_{t-1}, \Gamma\right) \propto p\left(\phi_{t} \mid \phi_{t-1}\right) p\left(\phi_{t} \mid \Gamma\right) .
$$

Hence, the prediction step of the particle filter can be seen as a two-step process. In the first place, samples from 
$p\left(\phi_{t} \mid \phi_{t-1}\right)$ follow a smooth motion model in order to enable the tracking of any possible motion, i.e. predictions are made according to

$$
\hat{\phi}_{t}=\phi_{t-1}+\eta\left(\sigma_{\phi}\right)
$$

where $\hat{\phi}_{t}$ denotes a predicted posture at time $t$ by the smooth motion model with standard deviation $\sigma_{\phi}$ empirically determined from training data.

In the second step, we prune those predictions $\hat{\phi}_{t}$ which are not accepted as feasible postures by our actionspecific model $p\left(\phi_{t} \mid \Gamma\right)$. For each rejected prediction $\hat{\phi}_{t}$, we resample from Eq.(5) until a feasible posture is generated. Finally, the new set of predicted particles $\phi_{t}$ is constituted by the accepted predictions $\hat{\phi}_{t} \cdot p\left(\phi_{t} \mid \Gamma\right)$ is defined as

$$
p\left(\phi_{t} \mid \Gamma\right)=\left\{\begin{array}{cc}
1 & \text { if }\left(\left|\tilde{\phi}_{t, d}-\tilde{g}_{j, d}\right|\right)<2 \sigma_{j, d}, \forall d=1 . . D \\
0 & \text { otherwise }
\end{array},\right.
$$

where the $D$-dimensional vectors $\tilde{\phi}_{t}$ and $\tilde{g}_{j}$ stand for the projections into the aSpace of $\phi_{t}$ and $\hat{g}_{j}$ respectively. $\hat{g}_{j}^{A}$ is the $j$-th posture from the mean performance computed for the action $A$ which probabilistically matched $\tilde{\phi}_{t}$ using a Gaussian conditional distribution with covariance derived from the training set. Finally, $\sigma_{j}=\left(\sigma_{j, 1}, \ldots, \sigma_{j, D}\right)$ stands for the learnt standard deviation of the $j$-th posture for the action $A$. Notice that the level of filtering depends on the number of $D$ dimensions considered when building the aspace representation.

By defining this filtering method, we aim to prune those predictions which are more distant than two times the learned standard deviation from the matched frame of the action model for a particular action. As a result, our dynamic model predicts feasible human postures avoiding particle wastage on postures which are not likely to appear during the performance of a particular action.

\section{Experimental results}

To test this work we used performances from a bending action. However, the approach is easily extensible to the remaining actions. Hence, we have tested the overall tracking approach for a sequence of a bending action not present in the training set used. It consisted in 72 frames from which we have 3D ground truth data available. The absolut position and orientation of the body were taken from ground truth data, and thereby, were not estimated by the tracker. Finally, we used a training set of 40 bending performances carried out by 9 different actors.

In order to enable the testing of the dynamic model without interferences derived from image-based likelihoods, we defined an ideally perfect likelihood function based on ground truth data. Given the 3D joint positions

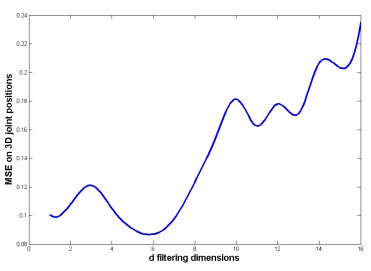

(a)

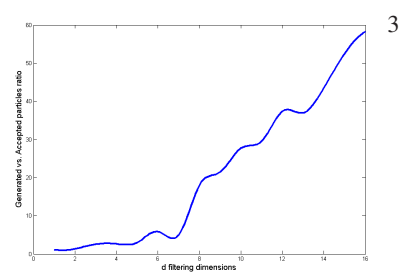

(b)

\section{Fig. 1. Error and particle rejection ratio de- pending on the number of $d$ dimensions.}

$\phi_{t}^{G T}$ from a sequence of ground truth data, and its corresponding 3D joint positions $\phi_{t}$ of the estimated postures from the tracker at time $t$, we define the likelihood of observing the image $I_{t}$ given the predicted posture $\phi_{t}$ as

$$
p\left(I_{t} \mid \phi_{t}\right) \propto e^{-\alpha \cdot \operatorname{dist}\left(\phi_{t}, \phi_{t}^{G T}\right)},
$$

where dist stands for the cumulative sum of Euclidean distance between each joint from the body model, and $\alpha$ is a scale factor which determines the "peakiness" of the likelihood function.

Subsequently, the learned bending action model was used in the prediction step of the tracker to filter postures which belong or not to the action model. We carried out several experiments using different number of $d$ dimensions to build the aSpace $\Omega_{d} . N=1000$ particles and $\alpha=5$ in the likelihood formulation where used in all the experiments. Fig.1.(a) shows the average MSE obtained on $3 \mathrm{D}$ joint positions for the final estimated postures computed as the expectation of the posterior pdf- against ground truth data varying the $d$ parameter. As $d$ increases, the action model gets more adapted to the training data. Hence, too low values for $d$ result in a poor filtering effect, since too many particles are accepted by the action model. On the other hand, too high values lead to overfitting to the training set, since the action model only accepts particles that are almost equal to postures used to learn the action model. For this experiment, we obtained better results for $d=6$ dimensions, meaning that a good compromise is achieved between generality of the model and non-feasible postures rejection. Alternatively, Fig.1.(b) shows the ratio of discarded particles vs. accepted particles by the action model for different values of $d$.

Then, we compared the results obtained using our action model against a smooth motion model without any filtering in order to test the effectiveness of the approach. We repeated the same experiment varying $N$ from 100 to 6000 particles, with $d=6$ and $\alpha=5$ for both the action model filtering and the smooth motion model approaches. In Fig.2 we observe the obtained MSE on final relative angles for the aSpace filtering method (solid line) and the simple smooth motion model (dashed line). We may observe that 


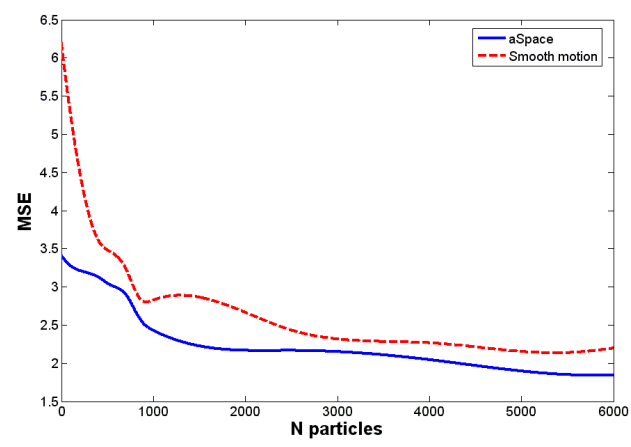

Fig. 2. MSE of both approaches.
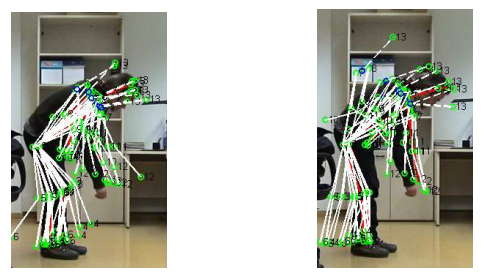

Fig. 3. Predictions of the aSpace (left) and smooth motion approaches (right).

the action model overperforms the smooth motion model in all the experiments. For instance, we obtain similar error measures using 1000 particles with the aSpace approach than 3000 particles without. Additionally, with very few particles, our approach never exceeds an error of 3.4 while the smooth motion model approach almost doubles this quantity. Hence, even with very few particles, our approach never totally looses the tracked object since it never produces non meaningful postures. This is depicted in Fig.3 where the frame 18 of the tracked sequence is plotted with a randomly selected set of predicted postures projected over it for the aspace approach, and the smooth motion model approach.

One may observe that the smooth motion model leads to unlikely and non feasible human postures for this action, while the aSpace filtering approach predicts natural and coherent human postures. Finally, some frames of the final estimated postures are shown in Fig.4 for $N=4000$ particles.

\section{Conclusion}

We have presented an efficient tracking approach based on particle filtering for full-body human tracking, which makes use of an action model to guide the prediction step of the particle filter. We compared the overall error of our approach against a smooth-motion model without
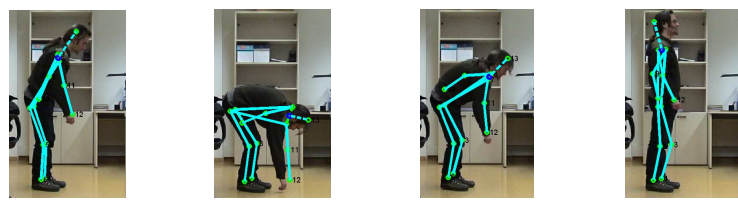

Fig. 4. Estimated frames 10, 30, 50 and 70.

filtering in the aSpace. Results point out that the action model approach drastically reduces the number of particles needed to track a 37 DOF human body model, thus reducing the high computational cost inherent to typical particle filtering methods. Moreover, given the PCA-like definition of the action space, the degree of dependence of the predictions to the training set can be tuned by considering more or less dimensions when building such space.

Future work relies on extending this approach to track transitions between actions. Furthermore, adding an image-based likelihood function we can result in a fully automatic tracking system. Finally, it is possible to improve the action model by considering other formulations which may improve the pruning effect providing more accuracy and efficiency to the overall tracking process.

Acknowledgments: This work has been supported by the Generalitat de Catalunya Research Department, by the EC grant IST-027110, by the Spanish MEC under projects TIC2003-08865 and DPI-2004-5414. J. Gonzàlez and J. Varona acknowledge the support of a Juan de la Cierva and Ramon y Cajal postdoctoral fellowships from the Spanish MEC, respectively.

\section{References}

[1] J. Deutscher and I. Reid. Articulated body motion capture by stochastic search. IJCV, 61(2):185-205, 2005.

[2] J. Gonzàlez. Human Sequence Evaluation: the Key-frame Approach. $\mathrm{PhD}$ thesis, Universitat Autònoma de Barcelona, 2004.

[3] J. Gonzàlez, J. Varona, X. Roca, and J.J. Villanueva. Analysis of human walking based on aSpaces. AMDO'04, September 2004

[4] M. Isard and A. Blake. Condensation - conditional density propagation for visual tracking. IJCV, 29(1):5-28, 1998.

[5] J. MacCormick and M. Isard. Partitioned sampling, articulated objects and interface-quality hand tracking. Dublin, 2000. ECCV.

[6] H. Ning, T. Tan, L. Wang, and W. Hu. Kinematics-based tracking of human walking in monocular video sequences. Image and Vision Computing, 22:429-441, 2004.

[7] I. Rius, D. Rowe, J. Gonzàlez, and X. Roca. A 3d dynamic model of human actions for probabilistic image tracking. In IbPRIA'2005.

[8] H. Sidenbladh, M.J. Black, and D.J. Fleet. Stochastic tracking of 3d human figures using $2 \mathrm{~d}$ image motion. In $E C C V$, volume 2, pages 702-718, Dublin, 2000.

[9] H. Sidenbladh, M.J. Black, and L. Sigal. Implicit probabilistic models of human motion for synthesis and tracking. In ECCV (1), pages 784-800, 2002. 